\title{
Investigation of Wire Coating Using Hydromagnetic Third-Grade Liquid for Coating along with Hall Current and Porous Medium
}

\author{
Zeeshan Khan $(\mathrm{D}){ }^{1}$ Haroon Ur Rasheed $\left(\mathrm{D},{ }^{2}\right.$ Wali Khan Mashwani ${ }^{\mathrm{D}},{ }^{3}$ Nasser Tairan, \\ Habib Shah $\mathbb{D}^{4}{ }^{4}$ Qayyum Shah, ${ }^{5}$ Waris Khan $\mathbb{D}^{3},{ }^{3}$ and Ikram Ullah ${ }^{6}$ \\ ${ }^{1}$ Sarhad University of Science and Information Technology, Peshawar, Khyber Pakhtunkhwa 25000, Pakistan \\ ${ }^{2}$ Department of Mathematics, Abdul Wali Khan University Mardan, Mardan, Khyber Pakhtunkhwa 23000, Pakistan \\ ${ }^{3}$ Institue of Numerical Sciences, Kohat University of Science and Technology, Kohat, Pakistan \\ ${ }^{4}$ College of Computer Science, Department of Computer Science, King Khalid University, Abha, Saudi Arabia \\ ${ }^{5}$ Department of Basic Science and Islamiat, University of Engineering and Technology, Peshawar, Pakistan \\ ${ }^{6}$ Department of Physics, Kohat Univesity of Science \& Technology, Kohat, Pakistan
}

Correspondence should be addressed to Wali Khan Mashwani; mashwanigr8@gmail.com

Received 12 December 2019; Accepted 1 April 2020; Published 27 July 2020

Academic Editor: Yannis Dimakopoulos

Copyright ( 92020 Zeeshan Khan et al. This is an open access article distributed under the Creative Commons Attribution License, which permits unrestricted use, distribution, and reproduction in any medium, provided the original work is properly cited.

\begin{abstract}
In this research study, we investigated and performed coating analysis of wire by using MHD convective third-order fluid in the presence of a permeable matrix taking into account the Hall current. The equations that control the motion of fluid in the chamber are first modeled and then numerically solved by using $4^{\text {th }}$ order Runge-Kutta-Fehlberg technique. The Runge-Kutta-Fehlberg method is a powerful tool used in this article to attain a numerical solution for a system of nonlinear ordinary differential equations describing the problem of fluid flow. The impact of governing parameters on velocity and temperature profiles is investigated graphically. It is noticed that the velocity profiles $u(r)$ rise as the value of viscoelastic parameter $\beta$ increases and slow down when the permeability parameter $K$ and the Hartmann number $M$ increase. Also, the temperature profiles $\theta(r)$ enhance as the Brinkman number $B r$, permeability parameter $K$, magnetic parameter $M$, and non-Newtonian parameter $\beta$ increase. For the sake of validation, the proposed method is also compared with BVPh2, and good agreement is found. Furthermore, a comparison is also done with the published work as a limiting case.
\end{abstract}

\section{Introduction}

Coating process is the major insulation of polymers with molten polymers for mechanical strength and to safeguard against the aggressive surroundings. Nylon, polysulfide, low-/high-density polyethylene (LDPE/HDPE), and plastic polyvinyl chloride (PVC) are common and important plastic resins used for wire coatings. The metallic coating technique is an engineering process use for the supply of insulation, environmental safety, mechanical damage and protect against signal attenuation. An appropriate and easy way of wire coating is the coaxial extrusion procedure that work at maximum pressure, temperature, and wire drawing velocity [1-4]. Pressure-type die has a very closed resemblance with an annulus, and accordingly, flow through this sort of die has a likeness with the flow through the annular region formed by the two coaxial cylinders, out of which the internal cylinder is moving in the axial direction, while the external cylinder is considered fixed. Distinct types of fluids are considered for wire, which depends upon the geometry of die, fluid viscosity, the temperature of the wire, and that of the molten polymer. A substantial care has been assumed to the Newtonian fluids to investigate the result of heat transfer analysis in the process of wire coating. Numerous fluids such as air, water, and other lubricants are considered as nonNewtonian fluids in science and engineering technologies. In many situations, the idea of the Newtonian fluid behavior is not so trivial, and it may be more complicated, and a perturbed (non-Newtonian) model must be considered. The non-Newtonian behavior exists in different liquid materials such as glue, paint, ketchup, custard, and blood. Because of its vast and significant uses in industry, petroleum and 
chemical engineering, and in other fluid mechanics, it obtained the attention of researchers [5-10].

Ellahi et al. [11] discussed the non-Newtonian micropolar liquid in the blood movement over composite stenosis. Eyring-Powell fluid is a non-Newtonian fluid, which was first presented in 1944 by Eyring-Powell. Many important features of Eyring-Powell fluid are discussed by researchers [12-16]. Wire coating technique is very important as it avoids injuries and decreases the losses that could be generated by machine oscillation. In manufacturing industries, various liquefy polymers are used in wire coating process. A melt polymer is put on over a wire, and then, the wire is dragged over the die covered by a viscoelastic material. Three methods used for wire coating are termed as electrostatistical deposition, dripping, and coaxial process. As compared to the remaining two, the dipping method in wire coating technique provides stronger links in the field, but it is very slow. It contains a payoff device, die, extruder device, cooling device, preheater, straightener, tester, capstan, and take-up roll. In the process, a bare wire is set rolling over the payoff device, which goes through straighter after which heat is provided to the wire by a preheater and cross-head die, which has a canonical die accumulating the liquefy polymer, and the wire is coated. The temperature of hot-coated wire is reduced with a cooling device after which it goes through a capstan, then a tester, and finally, the coated wire is puffing at take-up reel. Many other researchers [17-23] use various non-Newtonian materials for the wire coating process. In magnetohydrodynamic (MHD), the magnetic field induces the current, which have a major effect on the motion of the fluid material. In recent years, MHD has been an attractive area for researcher because of its vast usage in different industrial process just as magnetic material and glass manufacture processing. Many researchers [24-29] considered MHD as a current conducting liquid in the presence/absence of magnetic field and its consequences.

Fluid flux through a permeable medium has a countless significance for scientists because of its broad scope in engineering technology. Some of renowned permeable media are wood, carbonate rocks, and metal foams. Many researchers [30-34] paid attention to permeable media. Currently, a thin permeable layer is used in different domestic and industrial applications such as filters, batteries, fuel cell, and printing papers. The study of the heat convection in the non-Newtonian liquids got interest with time because of its usage in different industries. Rehman and Nadeem [35] investigated heat convective analysis for multidirectional stagnation flow movement. Khan along with other researchers [36-41] studied the effect of thermal convection and MHD fluid flow. By studying all the above studies, it is found that wire coating technique with MHD of a viscoelastic Eyring-Powell liquid as coating substance has yet not been discussed. In this paper, a procedure of the wire coating process with impact of thermal generation and a permeable medium along with temperature dependent flexible viscosity using Vogel's and Reynolds is discussed.

In this study, we consider the influence of MHD and heat transfer on the steady flow of a viscoelastic fluid, in which the wire is drawn at a higher speed in the presence of a porous medium and taking into account the Hall current. Although there are several studies of the flow and heat transfer of nonNewtonian fluids, a careful study of the literature shows that very little attention is paid to a viscoelastic fluid. As far as we know, no one has studied the MHD flow and heat transfer of a viscoelastic fluid to analyze wire coating in the presence of a porous medium. In this context, the governing equations for the velocity and temperature profiles are solved by the $4^{\text {th }}$ order Runge-Kutta method.

\section{Mathematical Analysis}

In coating process, the wire is extruded in a central line of the die of the coating chamber along with speed $V$ having fluid temperature $\theta_{W}$ and radius of the wire being $R_{W}$. The bath of third-grade fluid utilized as a melt polymer, such as polyvinyl chloride (PVC), for coating purposes in the presence of porous media inside the static pressure driven type die of length $L$, radius $R_{d}$, and the temperature $\theta_{d}$ is shown in Figure 1 . The liquid performs on a constant axial pressure gradient, and magnetic field of strength $B_{0}$ applies in transverse. Magnetic field is applied perpendicular to the flow direction to an incompressible fluid. Reynolds number is taken small enough as possible so that the induced magnetic field can be ignored. The Lorentz force can affect this installation, and as a result, the coating process gets affected from it.

The die is full from an incompressible fluid of the third grade. The wire and die are concentric, and in the center of the wire, a coordinate system is chosen, so that, $r$ is taken normal to the flow direction, while the $z$-axis is considered in the direction of stream. The flow is measured steady, laminar, and axisymmetric. In addition, the design of the coating matrix is more important as it greatly affects the quality of the final product. For this reason, a pressure stamp coating type is considered for the wire coating process. With the aforementioned reference frame and assumptions, the fluid velocity, extra stress tensor, and temperature fields are considered as follows:

$$
\begin{aligned}
w & =(0,0, u(r)), \\
S & =(r), \\
\theta & =\theta(r) .
\end{aligned}
$$

Boundary conditions are

$$
\begin{aligned}
& u=V, \theta=\theta_{w} \text { at } r=R_{w}, \\
& u=0, \theta=\theta_{d} \text { at } r=R_{d} .
\end{aligned}
$$

For third-grade fluid, the extra stress tensor is defined as follows:

$$
\begin{aligned}
S= & \eta A_{1}+\alpha_{1} A_{2}+\alpha_{2} A_{1}+\tau_{1} A_{2}+\tau_{2}\left(A_{1} A_{2}+A_{2} A_{1}\right) \\
& +\tau_{3}\left(\operatorname{tr} A_{2}\right) A_{1},
\end{aligned}
$$

where $\eta=\eta(\theta)$ denotes the coefficient of viscosity $(\mathrm{kg} / \mathrm{ms})$. Here, $\alpha_{1}$ and $\alpha_{2}$ are the $2^{\text {nd }}$ order material constants $(\mathrm{kg} / \mathrm{m})$, and the signs $\tau_{1}, \tau_{2}$, and $\tau_{3}$ are the $3^{\text {rd }}$ order non-Newtonian constants $(\mathrm{kg} / \mathrm{ms})$ and $t r$ denotes the trace of the matrix.

The quantities $A_{n}$ denote Rivlin-Ericksen tensors, which are shown from the recursive formula as follows: 


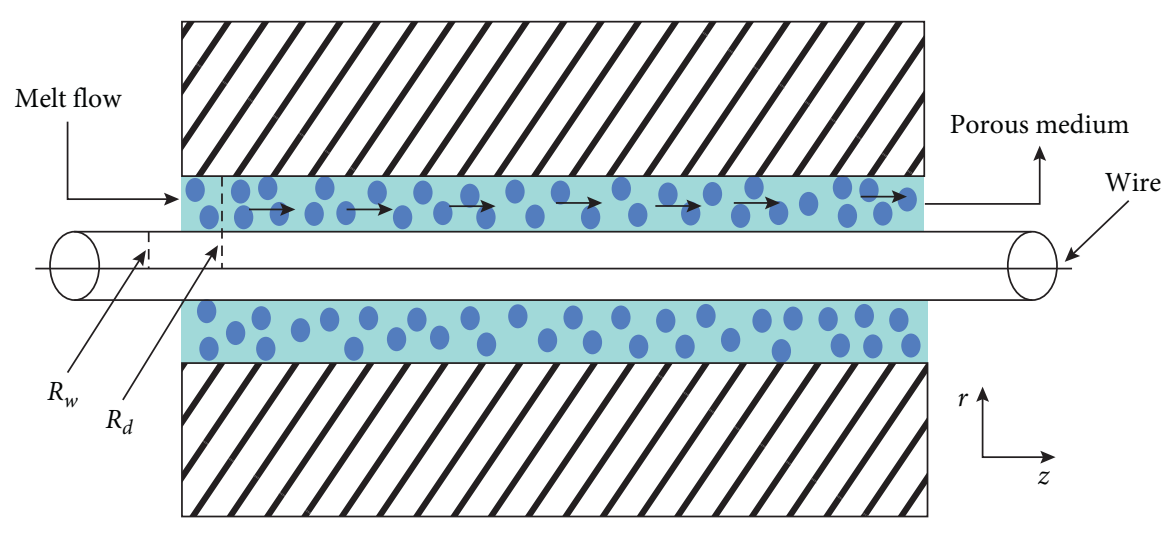

Figure 1: Geometry of the model problem.

$A_{1}=L^{T}+L, A_{n}=A_{n-1} L^{T}+L A_{n-1}+\frac{\mathrm{D} A_{n-1}}{\mathrm{D} t}, \quad n=2,2,3, \ldots$,

where $T$ denotes the matrix transpose.

2.1. Governing Equations. The governing equations such as the continuity, momentum, and the energy equations which particulates fluid flow of an incompressible third-grade fluid are given as follows [1-5]:

$$
\begin{aligned}
\nabla \cdot w & =0, \\
\rho \frac{\mathrm{D} u}{\mathrm{D} t} & =-\nabla p+F+J \times B-\frac{\eta u}{K}, \\
\rho c_{p} \frac{\mathrm{D} \theta}{\mathrm{D} t} & =k \nabla^{2} \theta+\phi,
\end{aligned}
$$

where $\rho$ denotes the density, $\mathrm{D} / \mathrm{D} t$ is the material derivative, $j$ represent the current density, $B$ denotes the magnetic field strength, $\eta$ is the dynamic viscosity of the fluid, $K$ is the permeability parameter, $C_{p}$ is the specific heat, $k$ is the thermal conductivity parameter, $\phi$ is the dissipation function, and $u$ is the fluid velocity.

Here, in equation (6), $j \times B$ denotes the body force per unit volume of electromagnetic origin, which appears because of the contact of the current and magnetic field. Electrostatic force due to charge density is considered to be small. A uniform magnetic field is applied in the positive radial direction normal to the wire, i.e., the retarding force per unit volume acting along the $z$-axis is given by

$$
J \times B=\left(0,0,-\sigma B_{0}^{2} u\right) .
$$

When the strength of magnetic field is very large, the generalized Ohm's law is modified to include the Hall current. If the Hall term is retained, the current density $j$ is given by

$$
J=\varepsilon\left[V \times B_{0}-\beta\left(J \times B_{0}\right)\right],
$$

where $\varepsilon$ is the electric conductivity of the fluid, and $\beta$ is the Hall factor. Equation (9) may be solved in $j$ to obtain the electromagnetic Lorentz's force in the following form:

$$
J \times B_{0}=-\frac{\varepsilon B_{0}^{2}}{m^{2}+1} V k
$$

where $m=\varepsilon \beta B_{0}$ is the Hall parameter, $B_{0}$ is the magnetic induction, and $k$ is the unit vector along the $z$-axis direction. Solving equation (10) for $j$, and substituting it in (6) and in view of equations (1)-(8), and assuming that there is no pressure gradient along the axial direction, we have the dimensional governing equation of the following form:

$$
\begin{gathered}
2\left(\tau_{2}+\tau_{3}\right) \frac{\mathrm{d}}{\mathrm{d} r}\left(r\left(\frac{\mathrm{d} u}{\mathrm{~d} r}\right)^{3}\right)+\frac{\eta}{r} \frac{\mathrm{d}}{\mathrm{d} r}\left(r \frac{\mathrm{d} u}{\mathrm{~d} r}\right)-\frac{\varepsilon B_{0}^{2}}{m^{2}+1}-\frac{\eta u}{K}=0, \\
k\left(\frac{\mathrm{d}^{2} \theta}{\mathrm{d} r^{2}}+\frac{1}{r} \frac{\mathrm{d} \theta}{\mathrm{d} r}\right)+\eta\left(\frac{\mathrm{d} u}{\mathrm{~d} r}\right)^{2} 2\left(\tau_{2}+\tau_{3}\right)\left(\frac{\mathrm{d} u}{\mathrm{~d} r}\right)^{4}=0
\end{gathered}
$$

Here, we have introduced some dimensionless parameters given by

$$
\begin{aligned}
r^{*} & =\frac{r}{R_{w}}, \\
u^{*} & =\frac{u}{V}, \\
\tau_{0} & =\tau_{2}+\tau_{3}, \\
\frac{R_{d}}{R_{w}} & =\delta>1, \\
\beta & =\frac{\tau_{0}}{\eta\left(R_{w}^{2} / V^{2}\right)}, \\
M^{2} & =\frac{\sigma B_{0}^{2} R_{w}}{\eta}, \\
K & =\frac{R_{w}^{2}}{V K^{*}}, \\
B r & =\frac{\eta V^{2}}{k\left(\theta_{d}-\theta_{w}\right)}, \\
\theta & =\frac{\theta-\theta_{w}}{\theta_{d}-\theta_{w}} .
\end{aligned}
$$


In light of equation (13), equations (2), (11), and (12) take the following form:

$$
\begin{aligned}
r \frac{\mathrm{d}^{2} u}{\mathrm{~d} r^{2}}+\frac{\mathrm{d} u}{\mathrm{~d} r}+2 \beta\left(3 r \frac{\mathrm{d}^{2} u}{\mathrm{~d} r^{2}}\left(\frac{\mathrm{d} u}{\mathrm{~d} r}\right)^{2}+\left(\frac{\mathrm{d} u}{\mathrm{~d} r}\right)^{3}\right) \\
-\left(\frac{M^{2}}{m^{2}+1}+K\right) u r=0, \\
\frac{\mathrm{d}^{2} \theta}{\mathrm{d} r^{2}}+\frac{1}{r} \frac{\mathrm{d} \theta}{\mathrm{d} r}+B r\left(\frac{\mathrm{d} u}{\mathrm{~d} r}\right)^{2}+2 B r \beta\left(\frac{\mathrm{d} u}{\mathrm{~d} r}\right)^{4}=0, \\
u(1)=1, \\
u(\delta)=0, \\
\theta(1)=0, \\
\theta(\delta)=1 .
\end{aligned}
$$

\section{Numerical Solution Procedure}

The higher order nonlinear governing differential equations given in equations (14) and (15) are first converted into ordinary first-order differential equations with the help of suitable transformation viewing in equation (19). They are solved numerically using the Runge-Kutta-Fehlberg method. First of all, we transform the equation of momentum and energy equations into a first-order form. Equations (14) and (15) become

$$
\begin{aligned}
& \frac{\mathrm{d}^{2} u}{\mathrm{~d} r^{2}}=\frac{\left[\left(\left(M^{2} / m^{2}+1\right)+K\right) u r-(\mathrm{d} u / \mathrm{d} r)-2 \beta(\mathrm{d} u / \mathrm{d} r)^{3}\right]}{\left[r+6 \beta r(\mathrm{~d} u / \mathrm{d} r)^{2}\right]} \\
& \frac{\mathrm{d}^{2} \theta}{\mathrm{d} r^{2}}=-\left[\frac{1}{r} \frac{\mathrm{d} \theta}{\mathrm{d} r}+B r\left(\frac{\mathrm{d} u}{\mathrm{~d} r}\right)^{2}+2 B r \beta\left(\frac{\mathrm{d} u}{\mathrm{~d} r}\right)^{4}\right]
\end{aligned}
$$

Defining new transforming variables for converting ordinary higher order differential equations into equations of first order:

$$
\begin{aligned}
u & =y_{1}, \\
u^{\prime} & =y_{2}, \\
u^{\prime \prime} & =y_{2}^{\prime}, \\
\theta & =y_{3}, \\
\theta^{\prime} & =y_{4}, \\
\theta^{\prime \prime} & =y_{4}^{\prime}
\end{aligned}
$$

Utilizing equation (19) in equations (17) and (18), we obtained the desired first-order differential equations given as follows:

$$
\begin{aligned}
& y_{2}^{\prime}=\frac{\left[\left(\left(M^{2} / m^{2}+1\right)+K\right) y_{1} r-y_{2}-2 \beta\left(y_{2}\right)^{3}\right]}{\left[r+6 \beta r\left(y_{2}\right)^{2}\right]}, \\
& y_{4}^{\prime}=-\left[\frac{1}{r} y_{4}+B r\left(y_{2}\right)^{2}+2 B r \beta\left(y_{2}\right)^{4}\right] .
\end{aligned}
$$

Boundary conditions given in equation (16) are converted to initial conditions as

$$
\begin{aligned}
& y_{1}(1)=1, \\
& y_{1}(\delta)=0, \\
& y_{3}(1)=0, \\
& y_{3}(\delta)=1 .
\end{aligned}
$$

\section{Validation of the Proposed Method}

To test our numerical solution, we make the following comparison, which confirms the carefulness of the proposed method. Our comparison is shown in Tables 1 and 2 for velocity and temperature profiles, respectively. Excellent agreement was noted between our data and previous published work by [21]. For more accuracy, the graphical comparison of the Runge-Kutta method and BVPh2 are also given in Figures 2 and 3, respectively.

\section{Discussion}

In this section, a brief study has been presented relevant to the emerging parameters which arises in the problem graphically and are discussed in Figures 4-12.

Figure 4 is sketched to examine the influence of magnetic parameter $M$ on the velocity profile $u(r)$. A clear decline in the velocity profile has been observed in the complete flow domain for greater values of magnetic parameter pointed out. This is due to the application of the magnetic field effect on an electrically conducting liquid which gives rise to opposing force recognized as the Lorentz force. This resistive force has propensity to slow down the motion of the fluid in the boundary layer section. Figure 5 depicts that as the magnitude of porosity parameter increases, as a result, the velocity profile decreases significantly. Figures 6 and 7 are designed to see the impact of the incompressible viscoelastic third-grade parameter $\beta$ and Hall current parameter $m$ on the velocity profile. It is noticed that both viscoelastic thirdgrade fluid parameter $\beta$ and Hall current parameter $m$ enhance the fluid velocity at all points in the presence/absence of magnetic field. We investigated the impact of the magnetic parameter $M$ upon the temperature profile $\theta(r)$ (Figure 8$)$. It is clear that the temperature profile grows up in the entire flow region of the fluid flow with increasing applied magnetic field $M$. This may be attributed to the boundary surface affects which override the effect of magnetic field.

The influence of porous parameter $K$ on the temperature profile $\theta(r)$ is shown in Figure 9. It revealed that the higher values of the porosity parameter $K$ heat up the fluid temperature uniformly in the flow region. In Figure 10, the impact of non-Newtonian parameter $\beta$ on the temperature has been analyzed. It is pointed out that a clear decline in the temperature of the fluid for higher values of the nonNewtonian parameter $\beta$ is observed. It is observed that the non-Newtonian parameter decreases the temperature both in the presence/absence of magnetic field. Figure 11 indicates that temperature distribution increases with an 
TABLE 1: Comparison of RK-4 and BVPh2 methods for velocity $u(r)$ profile with published work reported by [21].

\begin{tabular}{lccc}
\hline$u(r)$ & $\begin{array}{c}\text { Runge-Kutta } \\
\text { method }\end{array}$ & BVPh2 & Published work [21] \\
\hline 1.0 & 1.00000000000 & 1.00000000000 & 1.00000000000 \\
1.4 & 0.69133128205 & 0.69133128206 & 0.69133128211 \\
1.8 & 0.44146461538 & 0.44146461531 & 0.44146461531 \\
2.2 & 0.24891076923 & 0.24891076926 & 0.24891076918 \\
2.6 & 0.10622358974 & 0.10622358965 & 0.10622358960 \\
3.0 & 0.00000000000 & 0.00000000000 & 0.00000000000 \\
\hline
\end{tabular}

TABLE 2: Comparison of RK-4 and BVPh2 methods for velocity $\theta(r)$ profile with published work reported by [21].

\begin{tabular}{lccc}
\hline$\theta(r)$ & $\begin{array}{c}\text { Runge-Kutta } \\
\text { method }\end{array}$ & BVPh2 & Published work [21] \\
\hline 1.0 & 0.00000000000 & 0.00000000000 & 0.00000000000 \\
1.4 & 0.3076294200 & 0.3076294214 & 0.3076294210 \\
1.8 & 0.5609506277 & 0.5609506256 & 0.5609506267 \\
2.2 & 0.7595871849 & 0.7595871853 & 0.7595871786 \\
2.6 & 0.9047988740 & 0.9047988743 & 0.9047988745 \\
3.0 & 1.00000000000 & 1.00000000000 & 1.00000000000 \\
\hline
\end{tabular}

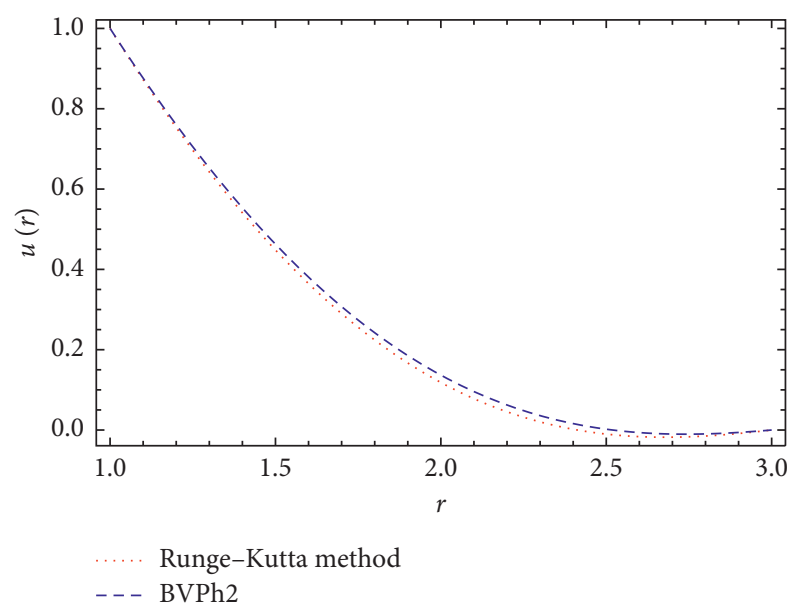

FIgURE 2: The comparison of RK-4 and BVPh2 methods for velocity $u(r)$ profile.

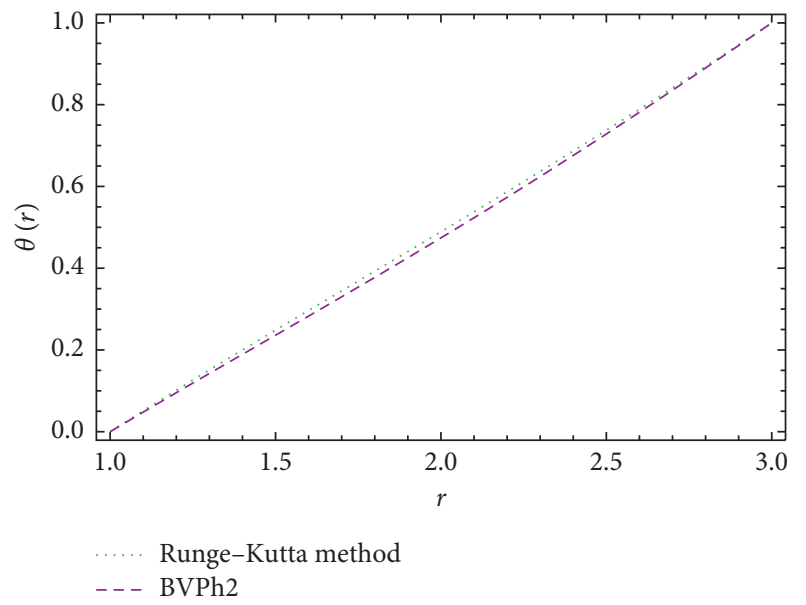

Figure 3: The comparison of RK-4 and BVPh2 methods for temperature $\theta(r)$ profile.

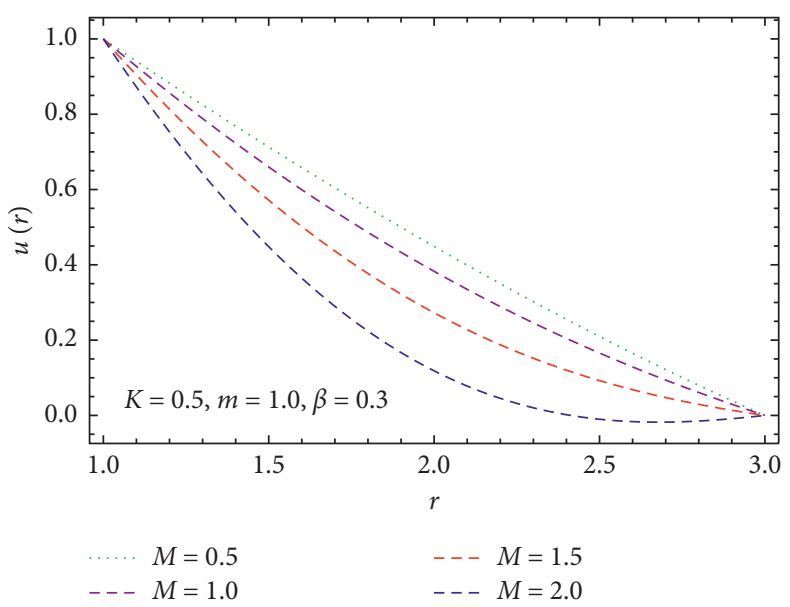

FIgURE 4: Impact of velocity profile $u(r)$ via $M$.

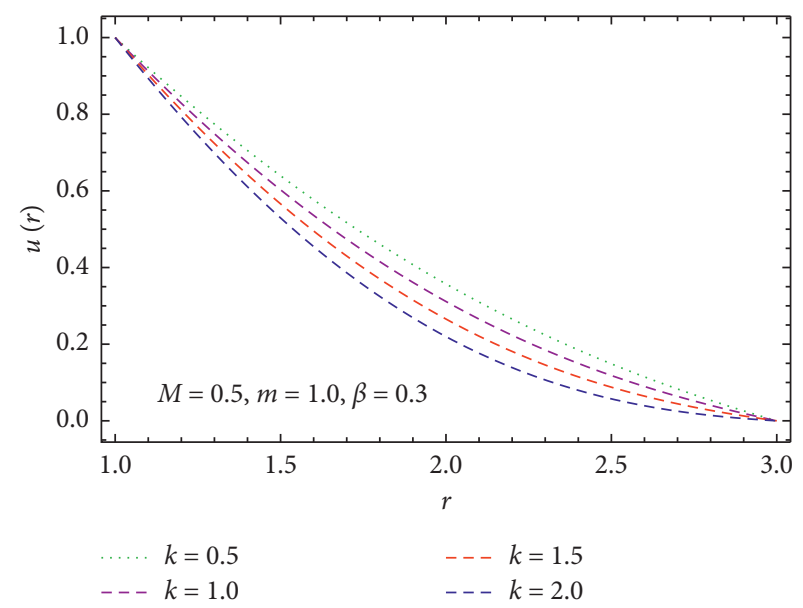

Figure 5: Impact of velocity profile $u(r)$ via $K$.

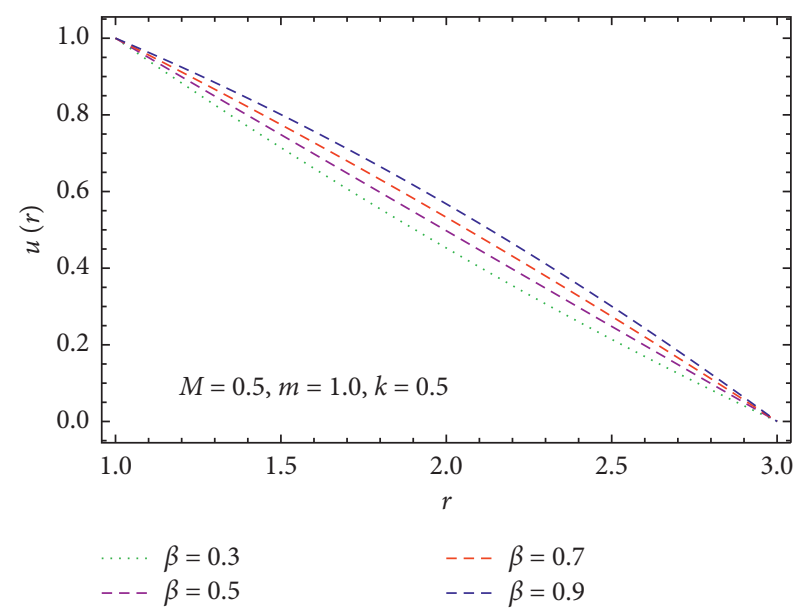

Figure 6: Impact of velocity profile $u(r)$ via $\beta$.

increase in the Brinkman number Br. It is seen that as the Brinkman number increases, the temperature profile increases significantly at all points. Hence, it is observed that in the process of wire coating, the Brinkman number is the relative measure of viscous heating with conducted heat, and 


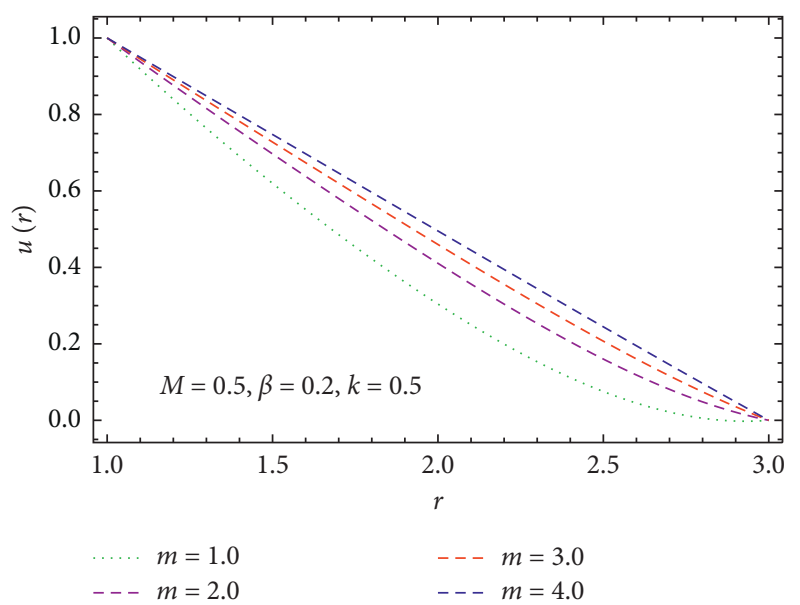

FIgURE 7: Impact of velocity profile $u(r)$ via $m$.

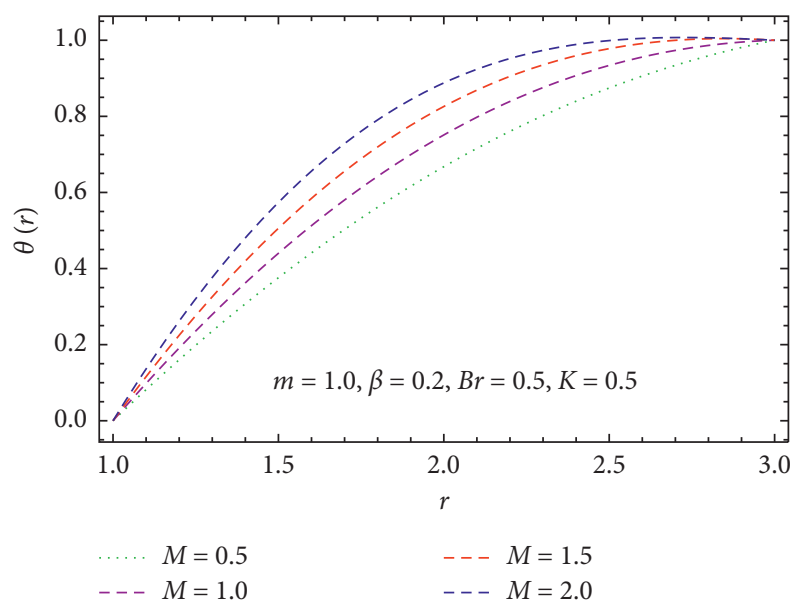

Figure 8: Impact of temperature profile $\theta(r)$ via $M$.

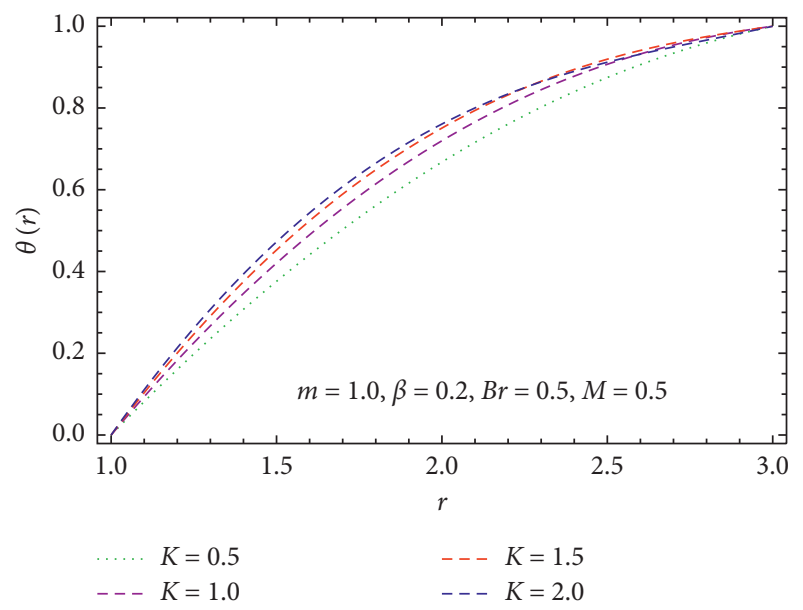

FIgURE 9: Impact of temperature profile $\theta(r)$ via $K$.

the temperature significantly accelerates uniformly at all points. Figure 12 shows that the Hall parameter decreases the temperature profiles in the presence of porous matrix.

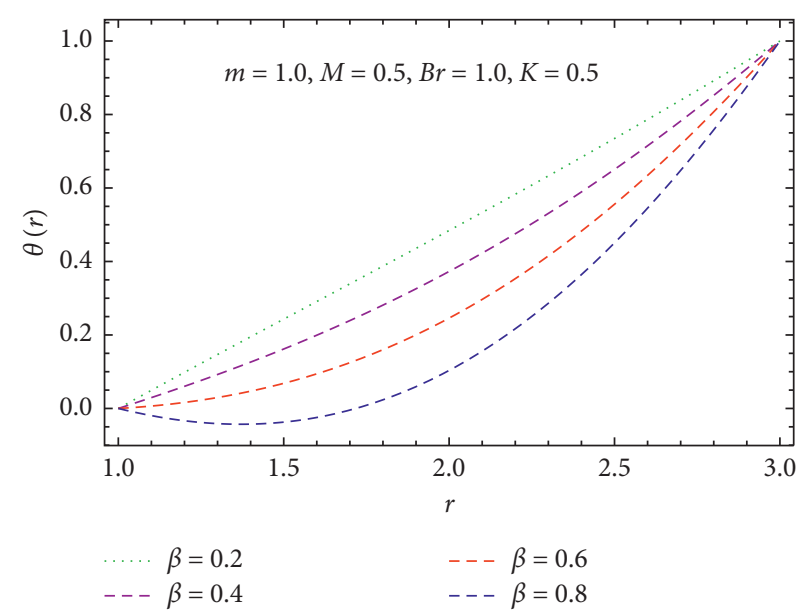

FIgURE 10: Impact of temperature profile $\theta(r)$ via $\beta$.

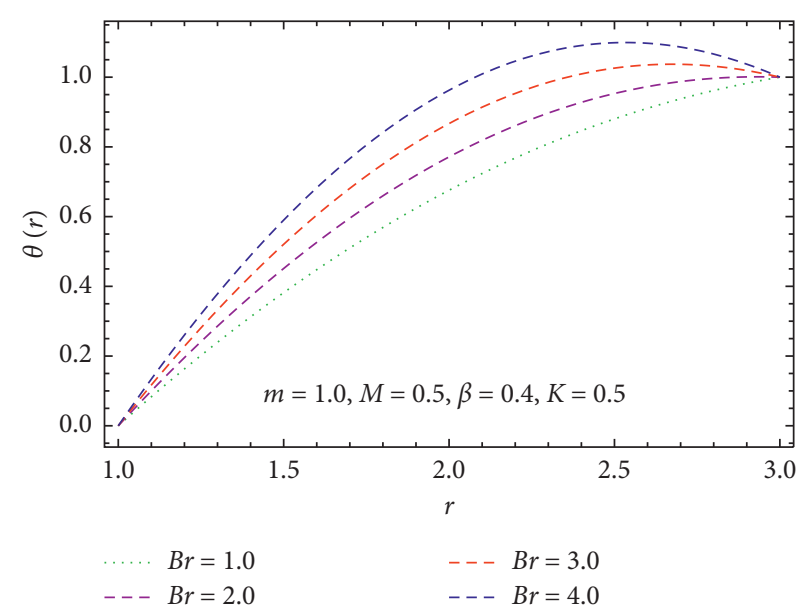

Figure 11: Impact of temperature profile $\theta(r)$ via $B r$.

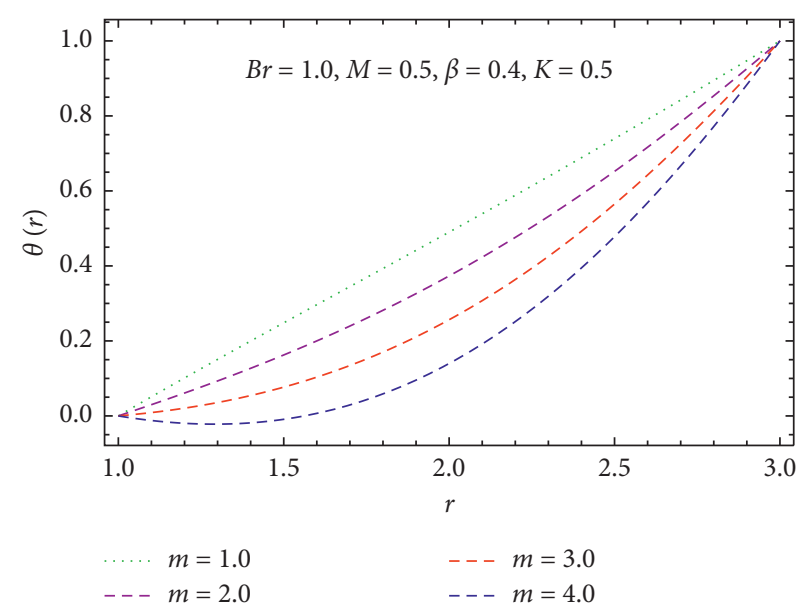

Figure 12: Impact of temperature profile $\theta(r)$ via $m$.

\section{Conclusions}

In this section, we have performed coating of wires by using incompressible viscoelastic third-grade fluid as a coating 
material for the wire as a melt polymer in the presence of pressure-type coating die. Expressions of velocity and temperature profiles are attained numerically with help of the Runge-Kutta-Fehlberg $4^{\text {th }}$ order method. For the validation of our numerical solution, a graphical and tabulated comparison has been done. The key points can be emphasized in the following bullets:

(i) The velocity profile increases with the increasing value of viscoelastic third-grade parameter

(ii) It is noticed that the velocity profiles grow up at high rate as the Hall parameter increases

(iii) The fluid temperature decreases with the rising values of the magnetic parameter, viscoelastic thirdgrade parameter, Hall parameter, and permeability parameter and increases with the Brinkman number

\section{Data Availability}

The data used to support the findings of this study are available from the corresponding author upon request.

\section{Conflicts of Interest}

The authors declare that they have no conflicts of interest.

\section{Acknowledgments}

The authors are thankful to King Khalid University of Saudi Arabia for financial support to this research under the grant number R.G.P.2/7/38.

\section{References}

[1] S. Akter and M. S. J. Hashmi, "Analysis of polymer flow in a conical coating unit: a power law approach," Progress in Organic Coatings, vol. 37, no. 1-2, pp. 15-22, 1999.

[2] S. Akter and M. S. J. Hashmi, "Plasto-hydrodynamic pressure distribution in a tepered geometry wire coating unit," in Proceedings of the 14th Conference of the Irish manufacturing committee (IMC14), pp. 331-340, Dublin, Ireland, September 1997.

[3] A. M. Siddiqui, T. Haroon, and H. Khan, "Wire coating extrusion in a pressure-type die in the flow of a third-grade fluid," International Journal of Nonlinear Sciences and Numerical Simulation, vol. 10, no. 2, pp. 247-257, 2009.

[4] R. T. Fenner and J. G. Williams, "Analytical methods of wire coating die design," Transactions and Journal of the Plastics Institute, vol. 35, pp. 701-706, 1967.

[5] S. Nadeem, S. Ahmad, and N. Muhammad, "CattaneoChristov flux in the flow of a viscoelastic fluid in the presence of Newtonian heating," Journal of Molecular Liquids, vol. 237, pp. 180-184, 2017.

[6] R. Tabassum, R. Mehmood, and S. Nadeem, "Impact of viscosity variation and micro rotation on oblique transport of $\mathrm{Cu}$-water fluid," Journal of Colloid and Interface Science, vol. 501, pp. 304-310, 2017.

[7] S. Nadeem and H. Sadaf, "Exploration of single wall carbon nanotubes for the peristaltic motion in a curved channel with variable viscosity," Journal of the Brazilian Society of $\mathrm{Me}$ chanical Sciences and Engineering, vol. 39, no. 1, pp. 117-125, 2017.
[8] I. Shahzadi, H. Sadaf, S. Nadeem, and A. Saleem, "Biomathematical analysis for the peristaltic flow of single wall carbon nanotubes under the impact of variable viscosity and wall properties," Computer Methods and Programs in Biomedicine, vol. 139, pp. 137-147, 2017.

[9] S. Ijaz, I. Shahzadi, S. Nadeem, and A. Saleem, "A clot model examination: with impulsion of nanoparticles under influence of variable viscosity and slip effects," Communications in Theoretical Physics, vol. 68, no. 5, p. 667, 2017.

[10] P. Kumam, Z. Shah, A. Dawar, H. Rasheed, and S. Islam, "Entropy generation in MHD radiative flow of CNTs Casson nanofluid in rotating channels with heat source/sink," Mathematical Problems in Engineering, vol. 2019, Article ID 9158093, 14 pages, 2019.

[11] R. Ellahi, S. U. Rahman, M. M. Gulzar, S. Nadeem, and K. Vafai, "A mathematical study of non-Newtonian micropolar fluid in arterial blood flow through composite stenosis," Applied Mathematics \& Information Sciences, vol. 8, no. 4, pp. 1567-1573, 2014.

[12] T. Hayat and S. Nadeem, "Aspects of developed heat and mass flux models on 3D flow of Eyring-Powell fluid," Results in Physics, vol. 7, pp. 3910-3917, 2017.

[13] T. Hayat and S. Nadeem, "Flow of 3D Eyring-Powell fluid by utilizing Cattaneo-Christov heat flux model and chemical processes over an exponentially stretching surface," Results in Physics, vol. 8, pp. 397-403, 2018.

[14] S. Ijaz and S. Nadeem, "A balloon model examination with impulsion of $\mathrm{Cu}$ nanoparticles as drug agent through stenosed tapered elastic artery," Journal of Applied Fluid Mechanics, vol. 10, no. 6, pp. 1773-1783, 2017.

[15] S. Ijaz and S. Nadeem, "A biomedical solicitation examination of nanoparticles as drug agents to minimize the hemodynamics of a stenotic channel," The European Physical Journal Plus, vol. 132, no. 11, p. 448, 2017.

[16] S. Saleem, S. Nadeem, and N. Sandeep, "A mathematical analysis of time dependent flow on a rotating cone in a rheological fluid," Propulsion and Power Research, vol. 6, no. 3, pp. 233-241, 2017.

[17] E. Mitsoulis, "Fluid flow and heat transfer in wire coating: a review," Advances in Polymer Technology, vol. 6, no. 4, pp. 467-487, 1986.

[18] E. B. Bagley and S. H. Storey, "Share rates and velocities of flow of polymers in wire-covering dies," Wire \& Wire Products, vol. 38, no. 8, p. 1104, 1963.

[19] N. A. Khan, F. Sultan, and N. A. Khan, "Heat and mass transfer of thermophoretic MHD flow of Powell-Eeyring fluid over a vertical stretching sheet in the presence of chemical reaction and Joule heating," International Journal of Chemical Reactor Engineering, vol. 13, no. 1, pp. 37-49, 2015.

[20] B. Mahanthesh, B. J. Gireesha, and R. S. R. Gorla, "Unsteady three-dimensional MHD flow of a nano Eyring-Powell fluid past a convectively heated stretching sheet in the presence of thermal radiation, viscous dissipation, and Joule heating," Journal of the Association of Arab Universities for Basic and Applied Sciences, vol. 23, no. 1, pp. 75-84, 2017.

[21] Z. Khan, A. K. Muhammad, S. Nasir, U. Murad, and S. Qayyum, "Solution of magnetohydrodynamic flow and heat transfer of radiative viscoelastic fluid with temperature dependent viscosity in wire coating analysis," PLoS One, vol. 13, no. 3, 2018.

[22] N. A. Khan and F. Sultan, "Homogeneous-heterogeneous reactions in an eyring-powell fluid over a stretching sheet in a porous medium," Special Topics \& Reviews in Porous Media: An International Journal, vol. 7, no. 1, pp. 15-25, 2016. 
[23] T. Hayat, N. Aslam, M. Rafiq, and F. E. Alsaadi, "Hall and Joule heating effects on peristaltic flow of Powell-Eyring liquid in an inclined symmetric channel," Results in Physics, vol. 7, pp. 518-528, 2017.

[24] Z. Khan, H. Rasheed, S. Islam, I. Khan, J. L. G. Guirao, and W. Khan, "Investigation of two-dimensional viscoelastic fluid with nonuniform heat generation over permeable stretching sheet with slip condition," Complexity, vol. 2019, Article ID 3121896, 8 pages, 2019.

[25] Z. Khan, H. U. Rasheed, T. A. Alkanhal, M. Ullah, I. Khan, and I. Tlili, "Effect of magnetic field and heat source on upperconvected-maxwell fluid in a porous channel," Open Physics, vol. 16, no. 1, pp. 917-928, 2018.

[26] S. Nadeem, S. Ahmad, N. Muhammad, and M. T. Mustafa, "Chemically reactive species in the flow of a Maxwell fluid," Results in Physics, vol. 7, pp. 2607-2613, 2017.

[27] Z. Khan, H. Ur Rasheed, M. Ullah, and T. Gul, "Analytical and numerical solutions of Oldroyd 8-constant fluid in doublelayer optical fiber coating fluid in double-layer optical fiber coating," Journal of Coatings Technology and Research, vol. 16, no. 1, pp. 235-248, 2018.

[28] A. U. Jan, R. Mehmood, and S. Nadeem, "Entropy analysis of radioactive rotating nanofluid with thermal slip," Applied Thermal Engineering, vol. 112, pp. 832-840, 2017.

[29] N. Muhammad and S. Nadeem, "Ferrite nanoparticles Ni$\mathrm{ZnFe} 2 \mathrm{O} 4, \mathrm{Mn}-\mathrm{ZnFe} 2 \mathrm{O} 4$ and $\mathrm{Fe} 2 \mathrm{O} 4$ in the flow of ferromagnetic nanofluid," The European Physical Journal Plus, vol. 132, no. 9, pp. 312-321, 2017.

[30] S. Ijaz and S. Nadeem, "Consequences of blood mediated nano transportation as drug agent to attenuate the atherosclerotic lesions with permeability impacts," Journal of Molecular Liquids, vol. 262, pp. 565-575, 2018.

[31] Z. Kkan, N. Tairan, W. K. Mashwani, H. Rasheed, H. Shah, and W. Khan, "MHD and slip effect on two-immiscible third grade fluid on thin film flow over a vertical moving belt," Open Phys, vol. 17, pp. 1-12, 2019.

[32] M. Rashid, I. Shahzadi, and S. Nadeem, "Corrugated walls analysis in microchannels through porous medium under Electromagnetohydrodynamic (EMHD) effects," Results in Physics, vol. 9, pp. 171-182, 2018.

[33] N. S. Akbar and S. Nadeem, "Double-diffusive natural convective peristaltic Prandtl flow in a porous channel saturated with a nanofluid," Heat Transfer Research, vol. 48, no. 4, pp. 283-290, 2017.

[34] I. Nadeem and S. Nadeem, "Impinging of metallic nanoparticles along with the slip effects through a porous medium with MHD," Journal of the Brazilian Society of Mechanical Sciences and Engineering, vol. 39, no. 7, pp. 2535-2560, 2017.

[35] F. U. Rehman and S. Nadeem, "Heat transfer analysis for three-dimensional stagnation-point flow of water-based nanofluid over an exponentially stretching surface," Journal of Heat Transfer, vol. 140, no. 5, p. 7, 2018.

[36] Z. Khan, H. U. Rasheed, I. Tlili, I. Khan, and T. Abbas, "Runge-Kutta $4^{\text {th }}$ order method analysis for viscoelastic Oldroyd 8-constant fluid used as coating material for wire with temperature dependent viscosity," Scientific Reports, vol. 8, no. 1, 2018.

[37] Z. Khan, W. A. Khan, H. Ur Rasheed, I. Khan, and K. S. Nisar, "Melting flow in wire coating of a third grade fluid over a die using Reynolds' and vogel's models with non-linear thermal radiation and joule heating," Materials, vol. 12, no. 19, p. 3074, 2019.

[38] Z. Khan, H. Ur Rasheed, S. O. Alharbi, I. Khan, T. Abbas, and D. Chin, "Manufacturing of double layer optical fiber coating using phan-thien-tanner fluid as coating material," Coatings, vol. 9, no. 2, p. 147, 2019.

[39] Z. Khan, M. Khan, S. Islam et al., "Analysis of magnetohydrodynamics flow and heat transfer of a viscoelastic fluid through porous medium in wire coating analysis," Mathematics, vol. 5, no. 2, p. 27, 2017.

[40] Z. Khan, R. Shah, S. Islam et al., "MHD flow and heat transfer analysis in the wire coating process using elastic-viscous," Coatings, vol. 7, no. 1, p. 15, 2017.

[41] H. Rasheed, Z. Khan, I. Khan, D. Ching, and K. Nisar, "Numerical and analytical investigation of an unsteady thin film nanofluid flow over an angular surface fluid flow over an angular surface," Processes, vol. 7, no. 8, p. 486, 2019. 